\title{
Mesophotic surveys of the flora and fauna at Johnston Atoll, Central Pacific Ocean
}

\author{
DANIEL WAGNER ${ }^{1}$, RANDALL K. KOSAKI ${ }^{1}$, HEATHER L. SPALDING ${ }^{2}$, ROBERT K. WHITTON ${ }^{3}$, \\ RICHARD L. PYLE ${ }^{3}$, ALISON R. SHERWOOD ${ }^{2}$, ROY T. TSUDA ${ }^{3}$ AND BARBARA CALCINAI ${ }^{4}$ \\ ${ }^{1}$ NOAA, Papahānaumokuākea Marine National Monument, 1845 Wasp Boulevard, Building 176, Honolulu, HI 96818, USA, \\ ${ }^{2}$ University of Hawai'i at Mānoa, Department of Botany, 3190 Maile Way, Honolulu, HI 96822, USA, ${ }^{3}$ Bernice P. Bishop Museum, \\ 1525 Bernice Street, Honolulu, HI 96817, USA, ${ }^{4}$ Universita Politecnica delle Marche, Dipartimento di Scienze della Vita e \\ dell'Ambiente, Via Brecce Bianche, 60131, Ancona, Italy
}

\begin{abstract}
Despite its extreme geographical isolation, numerous expeditions have surveyed the marine flora and fauna of Johnston Atoll. However, historical information about the marine biodiversity of Johnston is mostly limited to SCUBA surveys in shallowwaters $(<30 \mathrm{~m})$, and submersible observations in deeper waters $(100-500 \mathrm{~m})$. Extensive coral reefs, known as mesophotic coral ecosystems, exist between these two depth ranges at Johnston, but have remained largely unexplored. We used closedcircuit rebreathers to survey eleven sites at mesophotic depths $(32-78 \mathrm{~m})$ surrounding Johnston Atoll. A total of 130 species were recorded, including 99 species of fish, 15 species of corals, nine species of macroalgae, three species of echinoderms, three species of sponges and one species of squat lobster. Most species recorded during our mesophotic surveys have previously been recorded on shallow-water $(<30 \mathrm{~m})$ reefs at Johnston, with the exception of one black coral, one zoanthid, one squat lobster, two macroalgae, three sponges, and 22 fish, which represent new records for the atoll. As noted in previous studies, our surveys found a near absence of endemism, and recorded high proportions of species that are also known from the Hawaiian Archipelago. The similarity between the mesophotic biodiversity of Johnston Atoll and Hawai $i$ provides further support for the strong connectivity between these two locations highlighted in previous studies.
\end{abstract}

Keywords: closed-circuit rebreathers, mesophotic coral ecosystems, range expansion, technical diving

Submitted 24 April 2014; accepted 23 June 2014

\section{INTRDDUCTION}

Located in the Central Pacific over $800 \mathrm{~km}$ from the Hawaiian Islands, $1,450 \mathrm{~km}$ from the Line Islands, and 2,500 km from the Marshall Islands, Johnston Atoll is the most remote atoll on Earth. Despite its remoteness, numerous expeditions have targeted the atoll in order to explore and characterize its marine biodiversity (reviewed by Amerson \& Shelton, 1976; Lobel \& Lobel, 2008). In 1880, a vessel of the North Pacific Guano Company collected 27 fish species from Johnston, including five undescribed species, which were subsequently described by scientists at the United States National Museum (Smith \& Swain, 1882). In 1923, a partnership between the US Navy, the Bishop Museum and the Bureau of the Biological Survey (now US Fish and Wildlife Service) led to a series of expeditions to the north-western Hawaiian Islands, Wake Atoll and Johnston Atoll. As part of these expeditions, several scientists visited Johnston Atoll in 1923 aboard the US Navy minesweepers 'Tanager' and 'Whippoorwill' (Amerson \& Shelton, 1976; Olson, 1996). These two minesweepers returned to the atoll in 1924 along with other US navy ships. Although no scientific personnel joined that

Corresponding author:

D. Wagner

Email: Daniel.Wagner@noaa.gov expedition, biological specimens were collected for the Bishop Museum (Amerson \& Shelton, 1976). Descriptions of the biota of Johnston Atoll, obtained as part of these efforts by the Bishop Museum to study the biodiversity throughout the Central Pacific, were later published in separate papers focusing on invertebrates (Edmondson et al., 1925; Clark, 1949), fish (Fowler \& Ball, 1925), insects (Bryan, 1926; Chilson, 1953) and birds (Olson, 1996).

In the 1960 s and 1970s, the Smithsonian Institution led the Pacific Ocean Biological Survey Program, a research programme that aimed to increase the knowledge about various islands throughout the Central Pacific. As part of the programme, several biologists visited Johnston Atoll between 1963 and 1973, and although the major focus was on seabirds, other biota were also studied and inventoried (Amerson \& Shelton, 1976). Throughout the 1960s, the University of Hawai'i conducted a series of studies to measure the effects of dredging on the reef ecosystems of Johnston Atoll, and in particular on the prevalence of ciguatera around the atoll (Brock et al., 1965, 1966; Buggeln \& Tsuda, 1969).

In 1983, the Hawai'i Undersea Research Laboratory brought its manned submersible 'Makali'i' to Johnston in order to study the deep waters surrounding the atoll. A total of 35 submersible dives were performed to maximum depths of $500 \mathrm{~m}$, four of which were dedicated to geological studies (Keating, 1985), and the remainder centred on biological investigations (Randall \& Ralston, 1984; Agegian \& 
Abbott, 1985; Randall et al., 1985; Ralston et al., 1986; Chave \& Mundy, 1994; Chave \& Malahoff, 1998). In 2000, the Bishop Museum coordinated a major effort to inventory the shallowwater $(<30 \mathrm{~m})$ marine biota of Johnston as part of a survey of non-indigenous marine species of the atoll (Coles et al., 2001). Following these efforts, the US National Oceanic and Atmospheric Administration (NOAA) has regularly visited Johnston since 2004, in order to further characterize and monitor the shallow-water $\left(<_{30} \mathrm{~m}\right)$ algae, fish, corals and other invertebrates of the atoll (Brainard et al., 2005; NOAA, 2006; Lobel \& Lobel, 2008; Tsuda et al., 2010).

As a result of the multitude of scientific expeditions that have surveyed Johnston, there is ample information available on the marine biodiversity found in the shallow waters $(<30 \mathrm{~m})$ of the atoll (reviewed by Coles et al., 2001; Lobel \& Lobel, 2008). Additionally, numerous records exist on the flora and fauna found in the deeper waters $(100-500 \mathrm{~m})$ surrounding Johnston Atoll (Randall \& Ralston, 1984; Agegian \& Abbott, 1985; Randall et al., 1985; Ralston et al., 1986; Chave \& Mundy, 1994; Chave \& Malahoff, 1998). However, as in many regions around the world, little is known about the marine biodiversity found between these two depth ranges. This intermediate depth range hosts mesophotic coral ecosystems (MCEs), which are light-dependent coral reefs found below the depth limits of conventional SCUBA diving $(>30 \mathrm{~m})$ that extend to the deepest portion of the euphotic zone, which may be over $150 \mathrm{~m}$ in some oceanic regions with high water clarity like Johnston (Maragos \& Jokiel, 1986; Kahng \& Maragos, 2006; Kahng et al., 2010). The fixed upper depth limit of MCEs $(30 \mathrm{~m})$ corresponds to the depth limits of conventional SCUBA diving and does not represent a static ecological boundary (Kahng et al., 2014). As a result, shallow-water $(<30 \mathrm{~m})$ and mesophotic reefs $(>30 \mathrm{~m})$ can be quite similar in the distributions of their flora and fauna, particularly close to the depth boundary between these two ecosystems.
The only information on the mesophotic biodiversity of Johnston Atoll is derived from a limited number of surveys for reef fish at depths between 25 and $75 \mathrm{~m}$ (Kosaki, 1989; Kosaki et al., 1991). The purpose of this study was to survey this historically under-surveyed depth range at Johnston, in order to characterize and quantify the mesophotic flora and fauna of the atoll. Additionally, this study sought to compare the mesophotic biodiversity between Johnston Atoll and the Hawaiian Archipelago, because numerous previous studies have noted strong faunal and floral similarities between these two geographical areas (Gosline, 1955; Buggeln \& Tsuda, 1969; Bailey-Brock, 1976; Grigg, 1981; Grigg et al., 1981; Randall et al., 1985; Maragos \& Jokiel, 1986; Kosaki et al., 1991; Coles et al., 2001; Maragos et al., 2004; Tsuda et al., 2010). Due to these similarities, Johnston Atoll has been considered an important stepping stone for marine organisms reaching the Hawaiian Archipelago (Grigg, 1981; Grigg et al., 1981), an interpretation that is supported by both genetic (Rivera et al., 2004, 2011; Timmers et al., 2011) and oceanographic studies (Kobayashi, 2006) that demonstrate strong connectivity between these two regions.

\section{MATERIALS AND METHODS}

All dive surveys were performed using closed-circuit rebreathers on a research expedition to Johnston Atoll aboard the NOAA ship 'Hi'ialakai' in the summer of 2013 (HA-13-01). Dive sites were chosen using historical charts, as well as new multibeam data collected by the NOAA ship 'Hi' ialakai'. Chosen areas contained steep vertical drop-offs and hard substrate at depths between 32 and $78 \mathrm{~m}$. A total of eleven dive sites were surveyed around Johnston Atoll (Figure 1). During each survey, one diver identified and counted all large, conspicuous, diurnally-active fish to the lowest possible taxonomic level along a $25 \times 2 \mathrm{~m}$ belt transect

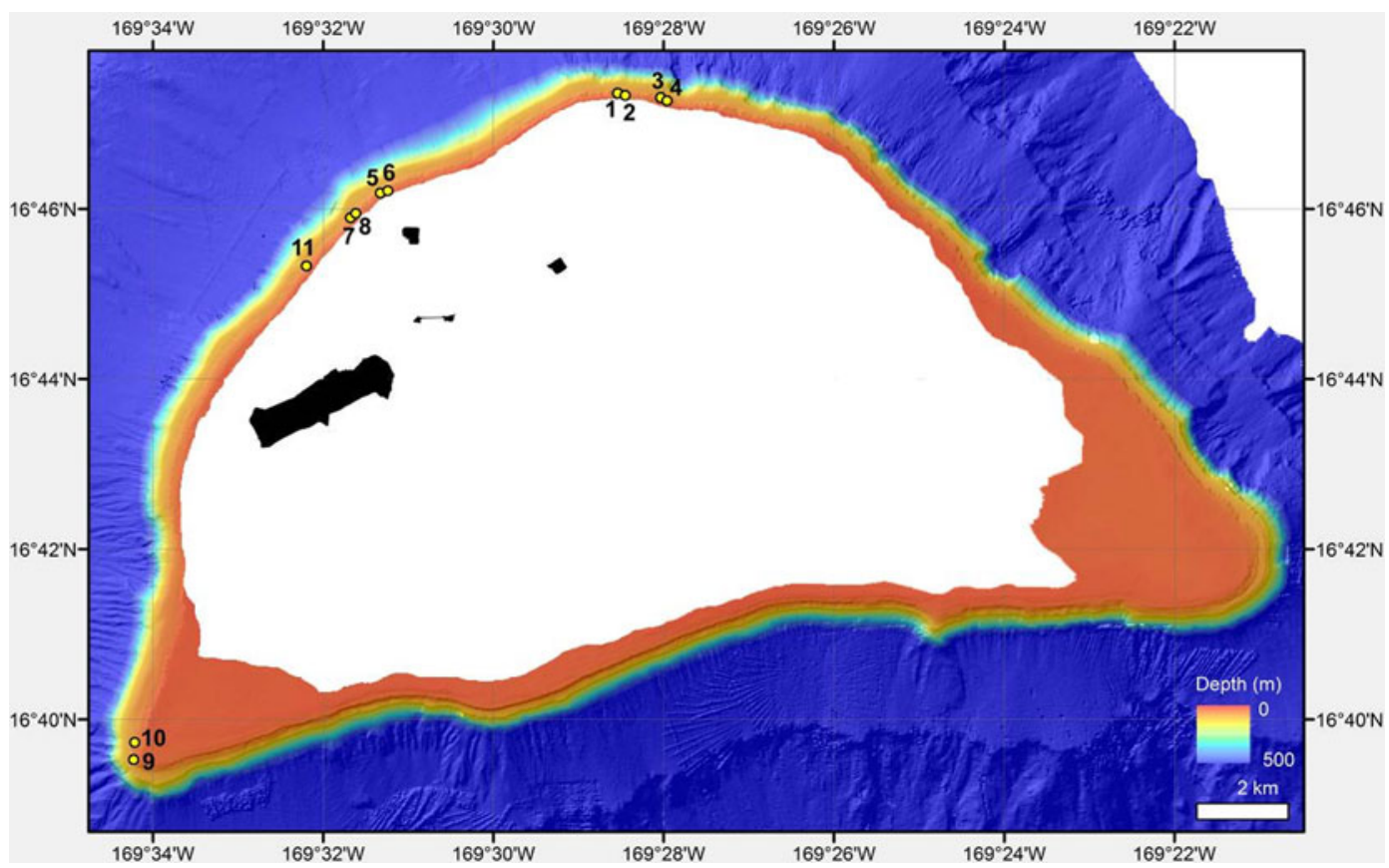

Fig. 1. Map showing the location of the eleven sites that were surveyed at mesophotic depths ( $32-78 \mathrm{~m})$ as part of surveys of the flora and fauna off Johnston Atoll. Areas in black show the four emergent land features of Johnston Atoll. 
(Kane et al., 2014). A second diver took photographs of the benthos at 25 randomly selected points along the transect using a $0.5 \mathrm{~m}^{2}$ photoquadrat. Upon transect completion, both divers collected macroalgae, sponges and corals that could not be identified in situ as time permitted. No crustose coralline algae or turf algae were collected due to time constraints. Collected samples were photographed in situ, placed into separate bags and preserved for later identification by taxonomy experts. Additionally, both divers recorded the presence of fish and macrobenthic species that were not captured during the transect itself, and documented observations with video vouchers. Benthic cover was determined from photoquadrat images with the aid of Coral Point Count with Excel extensions software (CPCe) using 100 random points per image (Kohler \& Gill, 2006). For this purpose, all macrobenthic organisms were identified to the lowest possible taxonomic level from photoquadrat images, using identifications of collected specimens by taxonomy experts where available. All species of the calcifying green alga Halimeda that were identified from collected specimens were grouped during the photoquadrat analysis, due to difficulties of differentiating individual species in photographs. Additionally, all identified organisms were classified post hoc based on their geographical distribution as being restricted to: (1) circumtropical or circumtemperate waters; (2) the Indo-Pacific; (3) the Indo-Pacific but not found in Hawai'i; or (4) Johnston Atoll and Hawai'i (Gosline, 1955; Randall et al., 1985; Kosaki et al., 1991).

\section{RESULTS}

\section{Macrobenthos}

A total of 31 macrobenthic species in seven phyla, 15 orders and 21 families were identified from mesophotic depths, including one antipatharian coral (Stichopathes sp.), one zoanthid (Palythoa caesia), three sponges (Dragmacidon sp.,
Prosuberites sp. and unidentified niphatid), two macroalgae (Neomeris vanbosseae and Halimeda distorta) and one squat lobster (Babamunida debrae) which were not previously recorded from Johnston Atoll (Table 1). Macrobenthic species identified to species level included 15 species of cnidarians, nine species of macroalgae, three species of echinoderms and one species of squat lobster (Table 1). With the exception of site 10 , which was mostly covered by crustose coralline algae $(58.0 \% \pm 22.7 \%)$, sponges $(25.5 \% \pm 15.9 \%)$ and corals $(16.5 \% \pm 20.6 \%)$, all other sites were dominated by sand, turf algae and crustose coralline algae, which collectively accounted for 68.8-93.4\% of the substrate (Figure 2). Macroalgae were generally the next most abundant macrobenthic group, and covered between 4.0 and $25.3 \%$ of the substrate, with the exception of site 10 where no macroalgae were recorded (Figure 2). Coral cover was generally low (0.56$4.56 \%$ ), with the exception of sites 4 and 10 , which had coral covers of $13.36 \%( \pm 27.6 \%)$ and $16.50 \%$ ( $\pm 20.6 \%)$, respectively (Figure 2). The most abundant macrobenthic species were the sponge Prosubirites sp. at site 10 $(17.19 \% \pm 14.1 \%)$, the green macroalga Caulerpa bikinensis at site $9(13.72 \% \pm 18.0)$, and the scleractinian corals Acropora cytherea $(10.72 \% \pm 28.5 \%)$ and Montipora capitata $(9.07 \% \pm 16.7 \%)$ at site 4 and 10 , respectively (Table 1$)$. Of the 31 macrobenthic species that were identified, 17 (54.8\%) are also found throughout other Indo-Pacific locations including Hawai'i; five (16.1\%) have widespread circumtropical or circumtemperate distributions, three (9.7\%) are found throughout the Indo-Pacific but not in Hawai' $i$, three (9.7\%) have unknown distributions, two $(6.5 \%)$ have a distribution restricted to Hawai'i and Johnston Atoll, and one (3.2\%; Caulerpa bikinensis) has a distribution restricted to Micronesia, French Polynesia and Johnston Atoll (Table 1).

\section{Fish}

A total of 99 fish species in 29 families were identified during mesophotic surveys, including 22 species which had not

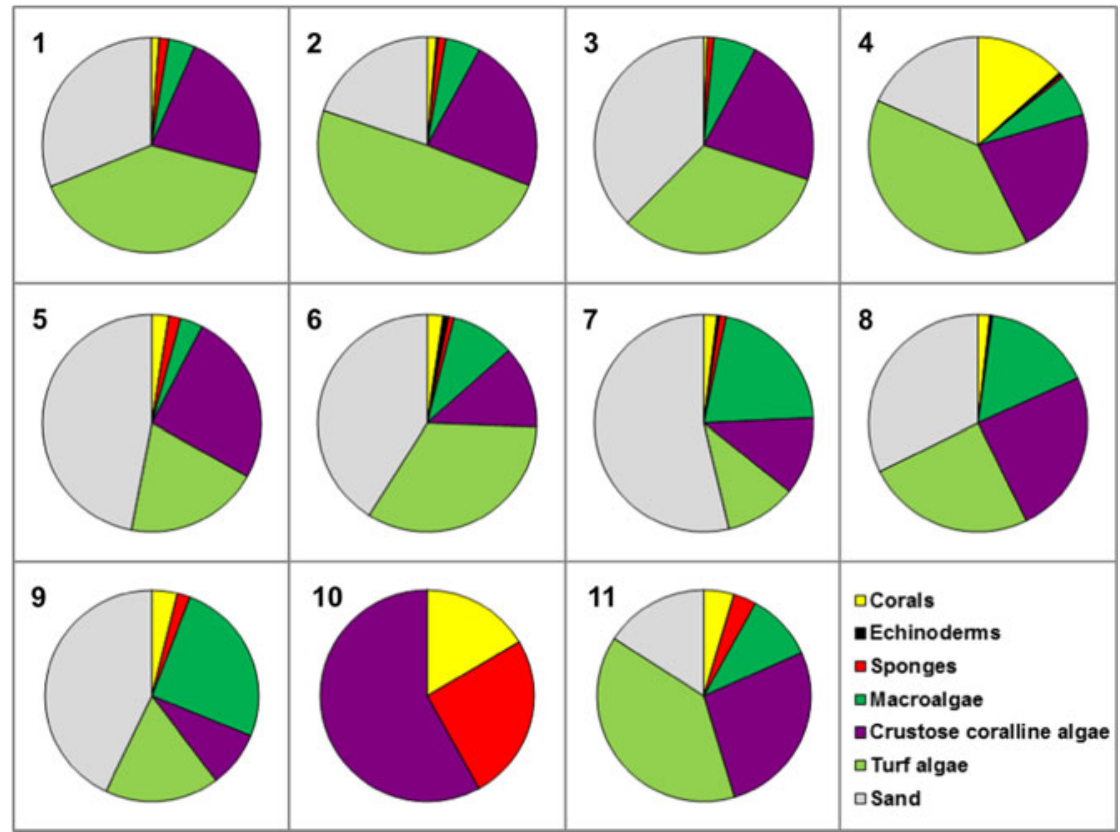

Fig. 2. Relative cover by macrobenthic group at the eleven sites that were surveyed at mesophotic depths (32-78 m) off Johnston Atoll. 


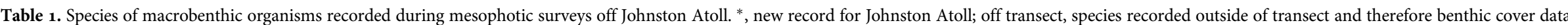
were not collected; ${ }^{\dagger}$, percentage cover at transect with the highest value; ${ }^{*}$, mean percentage cover of all transects \pm standard deviation.

\begin{tabular}{|c|c|c|c|c|c|c|c|c|c|}
\hline Phylum & Order & Family & Species & Authority & Depth (m) & $\begin{array}{l}\text { Identification } \\
\text { type }\end{array}$ & $\begin{array}{l}\text { Maximum } \\
\operatorname{cover}^{\dagger}(\%)\end{array}$ & $\begin{array}{l}\text { Mean cover } \\
\text { (\%) }\end{array}$ & $\begin{array}{l}\text { Geographical } \\
\text { distribution }\end{array}$ \\
\hline Arthropoda & Decapoda & Munididae & Babamunida debrae* & Baba, 2011 & 61 & Visual & Off transect & Off transect & Johnston and Hawai' $\mathrm{i}$ \\
\hline \multirow[t]{16}{*}{ Cnidaria } & Anthothecata & Milleporidae & Millepora tenera & Boschma, 1949 & $46-64$ & Visual & 1.25 & $0.12( \pm 0.38)$ & Indo-Pacific not Hawai $\mathrm{i}$ \\
\hline & Anthothecata & Stylasteridae & Distichopora violacea & (Pallas, 1766) & $64-70$ & Visual & 6.70 & $0.66( \pm 2.00)$ & Indo-Pacific not Hawai $\mathrm{i}$ \\
\hline & Antipatharia & Antipathidae & Antipathes griggi & Opresko, 2009 & 76 & Specimen & Off transect & Off transect & Johnston and Hawai'i \\
\hline & Antipatharia & Antipathidae & Cirrhipathes cf. anguina & (Dana, 1846) & 47 & Specimen & 0.30 & $0.03( \pm 0.09)$ & Indo-Pacific \\
\hline & Antipatharia & Antipathidae & Stichopathes sp.* & $\mathrm{N} / \mathrm{A}$ & 61 & Specimen & Off transect & Off transect & Indo-Pacific \\
\hline & Antipatharia & Myriopathidae & Myriopathes cf. ulex & (Ellis \& Solander, 1786) & 61 & Specimen & Off transect & Off transect & Indo-Pacific \\
\hline & Scleractinia & Acroporidae & Acropora cytherea & (Dana, 1846$)$ & $46-67$ & Visual & 10.72 & $1.01( \pm 3.22)$ & Widespread \\
\hline & Scleractinia & Acroporidae & Montipora capitata & (Dana, 1846) & $46-70$ & Visual & 9.07 & $2.06( \pm 2.55$ & Indo-Pacific \\
\hline & Scleractinia & Agariciidae & Leptoseris incrustans & (Quelch, 1886) & $46-67$ & Visual & 0.62 & $0.08( \pm 0.18)$ & Indo-Pacific \\
\hline & Scleractinia & Agariciidae & Leptoseris tubulifera & Vaughan, 1907 & $64-67$ & Visual & 0.29 & $0.04( \pm 0.09$ & Indo-Pacific \\
\hline & Scleractinia & Agariciidae & Leptoseris hawaiiensis & Vaughan, 1907 & $46-70$ & Visual & 0.73 & $0.17( \pm 0.26)$ & Indo-Pacific \\
\hline & Scleractinia & Agariciidae & Pavona maldivensis & (Gardiner, 1905) & 67 & Visual & 0.20 & $0.02( \pm 0.06)$ & Indo-Pacific \\
\hline & Scleractinia & Fungidae & Cycloseries vaughani & (Boschma, 1923) & $46-66$ & Visual & 0.18 & $0.03( \pm 0.09)$ & Indo-Pacific \\
\hline & Scleractinia & Poritidae & Porites lobata & Dana, 1846 & $46-67$ & Visual & 0.88 & $0.30( \pm 0.27)$ & Indo-Pacific \\
\hline & Zoantharia & Sphenopidae & Palythoa caesia* & Dana, 1846 & 46 & Visual & 0.08 & $0.01( \pm 0.02)$ & Indo-Pacific \\
\hline & & & Total cnidarians & & & & 16.50 & $4.54( \pm 5.31)$ & \\
\hline \multirow[t]{4}{*}{ Echinodermata } & Camarodonta & Toxopneustidae & Tripneustes gratilla & (Linnaeus, 1758) & 46 & Visual & 0.13 & $0.02( \pm 0.04$ & Indo-Pacific \\
\hline & Diadematoida & Diadematidae & Diadema paucispinum & Agassiz, 1863 & $46-47$ & Visual & 0.80 & $0.13( \pm 0.25)$ & Indo-Pacific \\
\hline & Valvatida & Acanthasteridae & Acanthaster planci & (Linnaeus, 1758) & 66 & Visual & 0.38 & $0.03( \pm 0.11)$ & Indo-Pacific \\
\hline & & & Total echninoderms & & & & 0.80 & $0.18( \pm 0.26)$ & \\
\hline \multirow[t]{5}{*}{ Porifera } & Hadromerida & Suberitidae & Prosuberites sp.* & N/A & $46-70$ & Specimen & 17.19 & $2.02( \pm 5.04)$ & Unknown \\
\hline & Halichondrida & Axinellidae & Dragmacidon sp.* & $\mathrm{N} / \mathrm{A}$ & $64-67$ & Specimen & 0.05 & $0.02( \pm 0.02)$ & Unknown \\
\hline & Haplosclerida & Niphatidae & Unidentified sp.* & $\mathrm{N} / \mathrm{A}$ & 46 & Specimen & 0.09 & $0.01( \pm 0.03)$ & Unknown \\
\hline & N/A & N/A & Unidentified sponges & $\mathrm{N} / \mathrm{A}$ & $46-70$ & Visual & 8.29 & $1.46( \pm 2.37)$ & $\mathrm{N} / \mathrm{A}$ \\
\hline & & & Total sponges & & & & 25.48 & $3.51( \pm 7.34)$ & \\
\hline \multirow[t]{8}{*}{ Chlorophyta } & Bryopsidales & Caulerpaceae & Caulerpa bikinensis & W.R. Taylor, 1950 & $46-67$ & Specimen & 13.72 & $1.34( \pm 4.11)$ & Pacific not Hawai'i \\
\hline & Bryopsidales & Caulerpaceae & Caulerpa racemosa & (Forsskål) J. Agardh, 1873 & $12-61$ & Specimen & Off transect & Off transect & Widespread \\
\hline & Bryopsidales & Caulerpaceae & Caulerpa serrulata & (Forsskål) J. Agardh, 1837 & $45-47$ & Specimen & 0.61 & $0.13( \pm 0.23)$ & Widespread \\
\hline & Bryopsidales & Halimedaceae & Halimeda discoidea & Decaisne, 1842 & 46 & Specimen & N/A & N/A & Widespread \\
\hline & Bryopsidales & Halimedaceae & Halimeda distorta* & (Yamada) Hillis-Colinvaux, 1968 & 46 & Specimen & N/A & N/A & Indo-Pacific \\
\hline & Bryopsidales & Halimedaceae & Halimeda taenicola & W.R. Taylor, 1950 & $55-67$ & Specimen & N/A & N/A & Indo-Pacific not Hawai $\mathrm{i}$ \\
\hline & Bryopsidales & Halimedaceae & Halimeda spp. & N/A & $46-67$ & Visual & 20.95 & $8.13( \pm 6.02)$ & N/A \\
\hline & Dasycladales & Dasycladaceae & Neomeris vanbosseae* & M. Howe, 1909 & 46 & Visual & 0.84 & $0.08( \pm 0.25)$ & Indo-Pacific \\
\hline Ochrophyta & Dictyotales & Dictyotaceae & Dictyota ceylanica & Kützing, 1859 & $61-67$ & Specimen & 0.72 & $0.07( \pm 0.22)$ & Indo-Pacific \\
\hline \multirow[t]{2}{*}{ Rhodophyta } & Bonnemaisoniales & Bonnemaisoniaceae & Asparagopsis taxiformis & (Delile) Trevisan de Saint-Léon, 1845 & 55 & Specimen & Off transect & Off transect & Widespread \\
\hline & & & Total macroalgae & & & & 25.32 & $9.74( \pm 7.89)$ & \\
\hline \multirow[t]{3}{*}{ N/A } & N/A & N/A & Crustose coralline algae & & $46-70$ & Visual & 58.02 & $23.42( \pm 13.06)$ & N/A \\
\hline & N/A & N/A & Turf algae & & $46-67$ & Visual & 49.17 & $27.71( \pm 14.64)$ & N/A \\
\hline & N/A & N/A & Sand & & $46-67$ & Visual & 53.67 & $30.89( \pm 15.93)$ & N/A \\
\hline 7 phyla & 15 orders & 21 families & 31 species ( 8 new records) & & & & & & \\
\hline
\end{tabular}




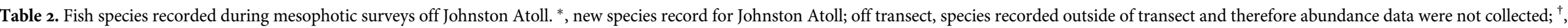
abundance at transect with the highest value; ${ }^{*}$, mean abundance of all transects \pm standard deviation; NR, depth not recorded.

\begin{tabular}{|c|c|c|c|c|c|c|}
\hline Family & Species & Authority & Depth (m) & $\begin{array}{l}\text { Maximum } \\
\text { abundance }^{\dagger}\left(/ \mathbf{m}^{2}\right)\end{array}$ & $\begin{array}{l}\text { Mean abundance } \\
\left(/ \mathbf{m}^{2}\right)\end{array}$ & $\begin{array}{l}\text { Geographical } \\
\text { distribution }\end{array}$ \\
\hline \multirow[t]{12}{*}{ Acanthuridae } & Acanthurus achilles & Shaw, 1803 & $8-45$ & Off transect & Off transect & Indo-Pacific \\
\hline & Acanthurus dussumieri & Valenciennes, 1835 & $62-70$ & 0.04 & $0.01( \pm 0.01)$ & Indo-Pacific \\
\hline & Acanthurus nigrofuscus* & (Forsskål, 1775) & 39 & Off transect & Off transect & Indo-Pacific \\
\hline & Acanthurus olivaceus & Bloch \& Schneider, 1801 & $36-67$ & 0.20 & $0.04( \pm 0.06)$ & Indo-Pacific \\
\hline & Acanthurus thompsoni & (Fowler, 1923) & $35-47$ & 0.40 & $0.04( \pm 0.12)$ & Indo-Pacific \\
\hline & Ctenochaetus hawaiiensis & Randall, 1955 & $47-66$ & 0.04 & $0.01( \pm 0.02)$ & Indo-Pacific \\
\hline & Ctenochaetus strigosus & (Bennett, 1828) & 47 & 0.08 & $0.01( \pm 0.02)$ & Johnston and Hawai'i \\
\hline & Naso caesius* & Randall \& Bell, 1992 & $46-59$ & Off transect & Off transect & Indo-Pacific \\
\hline & Naso hexacanthus & (Bleeker, 1855) & $11-66$ & 0.08 & $0.01( \pm 0.03)$ & Indo-Pacific \\
\hline & Naso lituratus & (Forster, 1801) & $33-70$ & 0.08 & $0.01( \pm 0.03)$ & Indo-Pacific \\
\hline & Naso thynnoides* & (Cuvier, 1829) & $48-70$ & Off transect & Off transect & Indo-Pacific \\
\hline & Zebrasoma flavescens & (Bennett, 1828) & $9-47$ & 0.04 & $0.01( \pm 0.01)$ & Indo-Pacific \\
\hline Apogonidae & Pristiapogon kallopterus & (Bleeker, 1856) & $65-78$ & Off transect & Off transect & Indo-Pacific \\
\hline Aulostomidae & Aulostomus chinensis & (Linnaeus, 1766) & $7-70$ & 0.02 & $0.00( \pm 0.01)$ & Indo-Pacific \\
\hline \multirow[t]{4}{*}{ Balistidae } & Melichthys vidua & (Richardson, 1845) & $9-70$ & 0.08 & $0.01( \pm 0.02)$ & Indo-Pacific \\
\hline & Sufflamen bursa & (Bloch \& Schneider, 1801) & $46-70$ & 0.08 & $0.02( \pm 0.03)$ & Indo-Pacific \\
\hline & Xanthichthys auromarginatus & (Bennett, 1832) & $21-65$ & 0.02 & $0.00( \pm 0.01)$ & Indo-Pacific \\
\hline & Xanthichthys caeruleolineatus* & Randall, Matsuura \& Zama, 1978 & $41-63$ & Off transect & Off transect & Indo-Pacific \\
\hline \multirow[t]{7}{*}{ Carangidae } & Carangoides orthogrammus & (Jordan \& Gilbert, 1882) & $10-66$ & 0.04 & $0.00( \pm 0.01)$ & Indo-Pacific \\
\hline & Caranx lugubris & Poey, 1860 & $50-66$ & 0.04 & $0.01( \pm 0.02)$ & Widespread \\
\hline & Caranx melampygus & Cuvier, 1833 & 46 & 0.02 & $0.00( \pm 0.01)$ & Indo-Pacific \\
\hline & Caranx sexfasciatus & Quoy \& Gaimard, 1825 & 67 & Off transect & Off transect & Widespread \\
\hline & Decapterus macarellus & (Cuvier, 1833) & 64 & Off transect & Off transect & Widespread \\
\hline & Naucrates ductor* & (Linnaeus, 1758) & 65 & Off transect & Off transect & Widespread \\
\hline & Uraspis helvola* & (Forster, 1801) & $60-70$ & Off transect & Off transect & Widespread \\
\hline \multirow[t]{2}{*}{ Carcharhinidae } & Carcharhinus amblyrhynchos & (Bleeker, 1856) & $46-70$ & 0.06 & $0.01( \pm 0.02)$ & Indo-Pacific \\
\hline & Triaenodon obesus & (Rüppell, 1837) & $50-70$ & 0.02 & $0.00( \pm 0.01)$ & Indo-Pacific \\
\hline \multirow[t]{9}{*}{ Chaetodontidae } & Chaetodon auriga & Forsskål, 1775 & $45-66$ & 0.02 & $0.00( \pm 0.01)$ & Indo-Pacific \\
\hline & Chaetodon kleinii* & Bloch, 1790 & 47 & 0.02 & $0.00( \pm 0.01)$ & Indo-Pacific \\
\hline & Chaetodon multicinctus & Garrett, 1863 & 42 & Off transect & Off transect & Johnston and Hawai' $\mathrm{i}$ \\
\hline & Chaetodon tinkeri & Schultz, 1951 & $47-72$ & 0.04 & $0.01( \pm 0.01)$ & Indo-Pacific \\
\hline & Chaetodon trifascialis & Quoy \& Gaimard, 1825 & 46 & 0.04 & $0.00( \pm 0.01)$ & Indo-Pacific \\
\hline & Forcipiger flavissimus & Jordan \& McGregor, 1898 & $42-46$ & 0.02 & $0.00( \pm 0.01)$ & Indo-Pacific \\
\hline & Forcipiger longirostris* & (Broussonet, 1782) & 32 & Off transect & Off transect & Indo-Pacific \\
\hline & Hemitaurichthys polylepis* & (Bleeker, 1857) & $31-32$ & Off transect & Off transect & Indo-Pacific \\
\hline & Hemitaurichthys thompsoni & Fowler, 1923 & 32 & Off transect & Off transect & Indo-Pacific \\
\hline \multirow[t]{3}{*}{ Cirrhitidae } & Cirrhitus pinnulatus & (Forster, 1801) & $61-67$ & Off transect & Off transect & Indo-Pacific \\
\hline & Paracirrhites arcatus & (Cuvier, 1829) & $9-67$ & Off transect & Off transect & Indo-Pacific \\
\hline & Paracirrhites forsteri & (Schneider, 1801) & 63 & Off transect & Off transect & Indo-Pacific \\
\hline Fistulariidae & Fistularia commersonii & Rüppell, 1838 & 67 & Off transect & Off transect & Indo-Pacific \\
\hline Gobiidae & Trimma milta* & Winterbottom, 2002 & 75 & Off transect & Off transect & Indo-Pacific \\
\hline
\end{tabular}


Table 2. Continued

\begin{tabular}{|c|c|c|c|c|c|c|}
\hline Family & Species & Authority & Depth (m) & $\begin{array}{l}\text { Maximum } \\
\text { abundance }{ }^{\dagger}\left(/ \mathbf{m}^{2}\right)\end{array}$ & $\begin{array}{l}\text { Mean abundance }{ }^{*} \\
\left(/ \mathbf{m}^{2}\right)\end{array}$ & $\begin{array}{l}\text { Geographical } \\
\text { distribution }\end{array}$ \\
\hline & Trimma taylori* & Lobel, 1979 & 61 & Off transect & Off transect & Indo-Pacific \\
\hline \multirow[t]{3}{*}{ Holocentridae } & Myripristis berndti & Jordan \& Evermann, 1903 & $35-67$ & Off transect & Off transect & Indo-Pacific \\
\hline & Myripristis chryseres & Jordan \& Evermann, 1903 & $58-67$ & Off transect & Off transect & Indo-Pacific \\
\hline & Sargocentron spiniferum & (Forsskål, 1775) & $32-67$ & 0.02 & $0.00( \pm 0.01)$ & Indo-Pacific \\
\hline \multirow[t]{12}{*}{ Labridae } & Bodianus albotaeniatus & (Valenciennes, 1839) & $45-67$ & 0.04 & $0.01( \pm 0.01)$ & Johnston and Hawai'i \\
\hline & Coris ballieui & Vaillant \& Sauvage, 1875 & 65 & Off transect & Off transect & Johnston and Hawai' $i$ \\
\hline & Coris flavovittata & (Bennett, 1828) & 47 & 0.02 & $0.00( \pm 0.01)$ & Johnston and Hawai' $\mathrm{i}$ \\
\hline & Coris gaimard & (Quoy \& Gaimard, 1824) & $34-46$ & 0.02 & $0.00( \pm 0.01)$ & Indo-Pacific \\
\hline & Labroides phthirophagus & Randall, 1958 & 50 & Off transect & Off transect & Johnston and Hawai' $\mathrm{i}$ \\
\hline & Iniistius pavo & (Valenciennes, 1840) & 65 & Off transect & Off transect & Indo-Pacific \\
\hline & Oxycirrhitus typus* & Bleeker, 1857 & 74 & Off transect & Off transect & Indo-Pacific \\
\hline & Oxycheilinus bimaculatus* & (Valenciennes, 1840) & 62 & Off transect & Off transect & Indo-Pacific \\
\hline & Pseudocheilinus evanidus & Jordan \& Evermann, 1903 & $36-70$ & 0.06 & $0.02( \pm 0.03)$ & Indo-Pacific \\
\hline & Pseudocheilinus ocellatus* & Randall, 1999 & $44-65$ & Off transect & Off transect & Indo-Pacific \\
\hline & Pseudojuloides cerasinus & (Snyder, 1904) & $45-46$ & 0.02 & $0.00( \pm 0.01)$ & Indo-Pacific \\
\hline & Thalassoma duperrey & (Quoy \& Gaimard, 1824) & $34-47$ & 0.04 & $0.00( \pm 0.01)$ & Johnston and Hawai'i \\
\hline Lethrinidae & Monotaxis grandoculis* & (Forsskål, 1775) & 34 & Off transect & Off transect & Indo-Pacific \\
\hline \multirow[t]{2}{*}{ Lutjanidae } & Aprion virescens & Valenciennes, 1830 & $46-60$ & 0.02 & $0.00( \pm 0.01)$ & Indo-Pacific \\
\hline & Aphareus furca & (Lacépède, 1801) & $7-75$ & Off transect & Off transect & Indo-Pacific \\
\hline Malacanthidae & Malacanthus brevirostris & Guichenot, 1848 & $42-50$ & 0.02 & $0.00( \pm 0.01)$ & Indo-Pacific \\
\hline Microdesmidae & Gunnellichthys curiosus* & Dawson, 1968 & $67-71$ & Off transect & Off transect & Indo-Pacific \\
\hline \multirow[t]{5}{*}{ Mullidae } & Mulloidichthys vanicolensis & (Valenciennes, 1831) & 32 & Off transect & Off transect & Indo-Pacific \\
\hline & Parupeneus cyclostomus & (Lacépède, 1801) & $8-72$ & 0.06 & $0.01( \pm 0.02)$ & Indo-Pacific \\
\hline & Parupeneus insularis* & Randall \& Myers, 2002 & $8-72$ & 0.02 & $0.00( \pm 0.01)$ & Indo-Pacific \\
\hline & Parupeneus multifasciatus & (Quoy \& Gaimard, 1825) & $34-67$ & 0.12 & $0.03( \pm 0.04)$ & Indo-Pacific \\
\hline & Parupeneus pleurostigma & (Bennett, 1831) & 47 & 0.02 & $0.00( \pm 0.01)$ & Indo-Pacific \\
\hline \multirow[t]{3}{*}{ Muraenidae } & Gymnothorax javanicus & (Bleeker, 1859) & $13-45$ & Off transect & Off transect & Indo-Pacific \\
\hline & Gymnothorax meleagris & (Shaw \& Nodder, 1795) & $45-64$ & 0.02 & $0.00( \pm 0.01)$ & Indo-Pacific \\
\hline & Gymnothorax undulatus & (Lacépède, 1803) & 60 & Off transect & Off transect & Indo-Pacific \\
\hline Ostraciidae & Ostracion whitleyi & Fowler, 1931 & $31-58$ & Off transect & Off transect & Indo-Pacific \\
\hline Pinguipedidae & Parapercis schauinslandii & (Steindachner, 1900) & $46-67$ & 0.03 & $0.01( \pm 0.02)$ & Indo-Pacific \\
\hline \multirow[t]{4}{*}{ Pomacanthidae } & Centropyge fisheri* & (Snyder, 1904) & $59-64$ & Off transect & Off transect & Indo-Pacific \\
\hline & Centropyge loricula & (Günther, 1874) & $37-70$ & 0.06 & $0.01( \pm 0.03)$ & Indo-Pacific \\
\hline & Centropyge nahackyi & Kosaki, 1989 & $41-70$ & 0.12 & $0.03( \pm 0.05)$ & Johnston and Hawai i \\
\hline & Centropyge potteri & (Jordan \& Metz, 1912) & 38 & Off transect & Off transect & Johnston and Hawai' $\mathrm{i}$ \\
\hline \multirow[t]{5}{*}{ Pomacentridae } & Apolemichthys arcuatus & (Gray, 1831) & $50-56$ & Off transect & Off transect & Johnston and Hawai'i \\
\hline & Chromis acares & Randall \& Swerdloff, 1973 & 47 & Off transect & Off transect & Indo-Pacific \\
\hline & Chromis agilis & Smith, 1960 & $61-75$ & Off transect & Off transect & Indo-Pacific \\
\hline & Chromis verater & Jordan \& Metz, 1912 & $45-78$ & 0.52 & $0.14( \pm 0.17)$ & Johnston and Hawai $\mathrm{i}$ \\
\hline & Dascyllus albisella & Gill, 1862 & $50-67$ & 0.02 & $0.00( \pm 0.01)$ & Johnston and Hawai $\mathrm{i}$ \\
\hline \multirow[t]{2}{*}{ Ptereleotridae } & Nemateleotris magnifica & Fowler, 1938 & $8-63$ & Off transect & Off transect & Indo-Pacific \\
\hline & Ptereleotris heteroptera & (Bleeker, 1855) & $62-64$ & Off transect & Off transect & Indo-Pacific \\
\hline \multirow[t]{2}{*}{ Scaridae } & Calotomus carolinus & (Valenciennes, 1840) & $7-70$ & Off transect & Off transect & Indo-Pacific \\
\hline & Calotomus sp.* & N/A (C. cf. spinidens or undescribed) & 64 & Off transect & Off transect & Johnston and Hawai'i \\
\hline
\end{tabular}



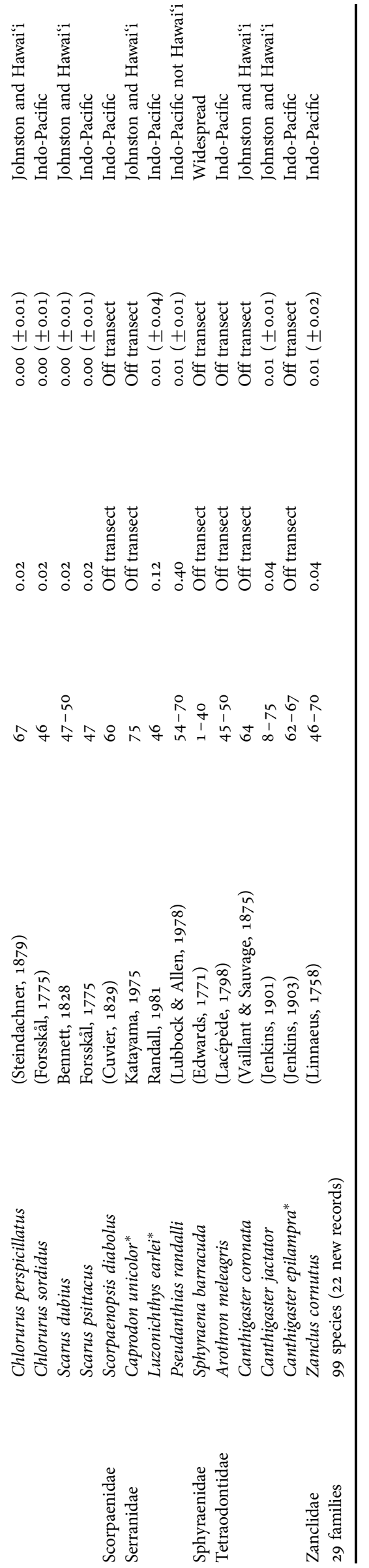

previously been recorded from Johnston Atoll (Table 2). Additionally, we recorded Centropyge fisheri, a species with an uncertain previous sighting at Johnston Atoll (Randall et al., 1985). In terms of number of individuals, Chromis verater, Pseudanthias randalli, Acanthurus olivaceus, A. thompsoni, Centropyge nahackyi, Parupeneus multifasciatus, Pseudocheilinus evanidus and Sufflamen bursa were most common (Table 2). With the exception of A. thompsoni, which was only recorded at one site, this group of fish was also most widespread, being recorded from seven (Chromis verater) to three (Pseudanthias randalli) of the surveyed sites. Among species that were less abundant, Canthigaster jactator, Parapercis schauinslandii, Centropyge loricula, Bodianus albotaeniatus, Chaetodon tinkeri, Ctenochaetus hawaiiensis, Zanclus cornutus and Acanthurus dussumieri were widespread on the surveyed mesophotic reefs, being recorded at three or more of the surveyed sites. Of the 99 fish species that were identified, $74(74.7 \%)$ are widely distributed throughout the Indo-Pacific including Hawai $i$, 18 (18.2\%) are restricted to Johnston Atoll and Hawai i, six (6.1\%) have widespread circumtropical distributions, and one (1.0\%; Pseudanthias randalli) is found throughout the Pacific but not in Hawai'i (Table 2).

\section{DISCUSSION}

In contrast to the shallow $(<30 \mathrm{~m})$ and deep-water $(100-$ $500 \mathrm{~m}$ ) marine biodiversity of Johnston, which has been extensively surveyed (reviewed by Coles et al., 2001), only limited surveys have been performed at mesophotic depths surrounding the atoll (Kosaki, 1989; Kosaki et al., 1991). This study thus represents the first dedicated effort to inventory and quantify the mesophotic flora and fauna at Johnston Atoll. We recorded a total of 130 species from mesophotic depths, most of which $(76.4 \%)$ have also been found during previous shallow-water $(<30 \mathrm{~m})$ surveys off Johnston Atoll (Tables 1 \& 2). This indicates that the mesophotic flora and fauna of Johnston is not composed of a specialized group of species, but rather consists of a subset of species that is also found in shallow waters $(<30 \mathrm{~m})$. The only species which have not been found in previous surveys off Johnston Atoll include one black coral, one zoanthid, one squat lobster, two macroalgae, three sponges and 22 fish (Tables 1 \& 2). With the exception of the three sponges, which have unknown geographical distributions (Table 1), all other species are commonly found in shallow-water reefs throughout the Indo-Pacific (Hoover, 2006; Huisman et al., 2007; Randall, 2007). Thus, it is likely that these species have been missed in previous shallow-water surveys at Johnston, and are not restricted to mesophotic depths at the atoll. This interpretation is consistent with most mesophotic surveys in the Hawaiian Islands, which indicate that the mesophotic flora and fauna are composed mainly of the same species, albeit in different proportions, that are also found in shallow waters (Brock \& Chamberlain, 1968; Parrish \& Boland, 2004; Rooney et al., 2010; Kane et al., 2014). However, in some Hawaiian locations, the mesophotic benthic flora and fauna appears distinct from shallow-water $(<30 \mathrm{~m})$ communities (Kahng \& Kelley, 2007; Spalding, 2012).

Our surveys indicate that mesophotic reefs off Johnston are covered mostly by turf algae, crustose coralline algae, macroalgae and sand (Figure 2). These groups were also identified as 
being most abundant in previous surveys on many shallowwater $(<18.3 \mathrm{~m})$ reefs off Johnston Atoll (Brainard et al., 2005; NOAA, 2006). However, previous shallow-water surveys off Johnston have also noted high coral cover on several reefs of the atoll, particularly inside the lagoon, where coral cover can approach 100\% (Maragos \& Jokiel, 1986; Jokiel \& Tyler, 1992; Brainard et al., 2005; NOAA, 2006). In contrast, coral cover at most of our mesophotic sites was generally low $(0.56-4.56 \%)$, with the exception of two sites which had $13.36 \%$ and $16.50 \%$ coral cover (Figure 1). The most commonly recorded corals during our mesophotic surveys were Acropora cytherea, Montipora capitata, Distichopora violacea and Millepora tenera (Table 1). With the exception of Distichopora violacea, these coral species are also dominant on shallow-water $(<18.3 \mathrm{~m})$ reefs of the atoll (Maragos \& Jokiel, 1986; NOAA, 2006). Additionally, Montipora patula, Pavona spp. and Pocillopora spp. are also dominant on shallow-water $\left(<_{18.3} \mathrm{~m}\right)$ reefs of Johnston (Maragos \& Jokiel, 1986; NOAA, 2006); all species which we did not record during our mesophotic surveys (Table 1). Among macrobenthic species identified during our mesophotic surveys, only the green alga Caulerpa bikinensis, the scleractinian coral Leptoseries hawaiiensis and the black corals Antipathes griggi and Myriopathes cf. ulex were recorded during previous deep-water $(>100 \mathrm{~m})$ submersible surveys off Johnston Atoll (Agegian \& Abbott, 1985; Maragos \& Jokiel, 1986; Coles et al., 2001).

The most common reef fish recorded during our mesophotic surveys were Chromis verater, Pseudanthias randalli, Acanthurus olivaceus, A. thompsoni, Centropyge nahackyi, Parupeneus multifasciatus, Pseudocheilinus evanidus and Sufflamen bursa (Table 2). While all of these species have been recorded during previous shallow-water $(<30 \mathrm{~m})$ surveys at Johnston, only Parupeneus multifasciatus and Sufflamen bursa are also reported as being abundant on shallow-water $\left(<_{18.3} \mathrm{~m}\right)$ reefs off the atoll (NOAA, 2006). Among species that were abundant at our mesophotic sites (Table 2), only Parupeneus multifasciatus was also reported as being abundant during previous deep-water $(100-365 \mathrm{~m})$ surveys at Johnston Atoll (Ralston et al., 1986). In contrast, many of the species we recorded at mesophotic depths have also been recorded during previous deep-water $\left(>_{100} \mathrm{~m}\right)$ submersible surveys off Johnston, including Carcharhinus amblyrhynchos, Myripristis chryseres, Parapercis schauinslandi, Carangoides orthogrammus, Caranx lugubris, C. melampygus, Chromis verater, Parupeneus cyclostomus, $P$. multifasciatus, Chaetodon tinkeri, Forcipiger flavissimus, Bodianus albotaeniatus, Acanthurus dussumeri, Naso hexacanthus, Zanclus cornutus and Xanthichthys auromarginatus (Randall et al., 1985; Ralston et al., 1986; Chave \& Mundy, 1994).

Abundances of macrobenthic organisms recorded during our surveys (Table 1), differed from those recorded during mesophotic surveys in the Hawaiian Islands (Rooney et al., 2010). The mesophotic benthos at Johnston consists mostly of crustose coralline algae $(8.6-58 \%)$, sand (o-54\%), turf algae $(0-49 \%)$ and macroalgae $(0-21 \%)$, with coral cover being low at most mesophotic locations (0.56-4.56\%) (Figure 2). In contrast, at similar depths (40-70 m) Rooney et al. (2010) noted that the macrobenthos in the north-western Hawaiian Islands was mostly covered by sand (45-55\%), macroalgae $(25-40 \%)$, crustose coralline algae $(\sim 5 \%)$, and coral $(\sim 2 \%)$. While the ultimate drivers for these differences remain unknown, they might be related to differences in the temperature regimes between Johnston Atoll and Hawaici. Johnston is located much closer to the equator $\left(16^{\circ} \mathrm{N}\right)$ than the Hawaiian Archipelago $\left(19-28^{\circ} \mathrm{N}\right)$ and as a result experiences elevated water temperatures. Previous studies note that sea surface temperatures show little seasonality at Johnston and range between 25 and $27^{\circ} \mathrm{C}$ (Ralston et al., 1986; Boehlert et al., 1992). In comparison, sea surface temperatures can dip as low as $16^{\circ} \mathrm{C}$ in the north-western Hawaiian Islands (Kane et al., 2014). Differences in temperature regime have been related to differences in macrobenthic cover within the Hawaiian Archipelago (Grigg et al., 2008; Rooney et al., 2010; Spalding, 2012), and might also explain why macroalgal cover is reduced at Johnston Atoll (0-21\%; Figure 2) compared to the north-western Hawaiian Islands (25-40\%; Rooney et al., 2010).

Consistent with previous biogeographical studies (Gosline, 1955; Maragos \& Jokiel, 1986; Kosaki et al., 1991; Maragos et al., 2004), our mesophotic surveys recorded a near absence of species that are endemic to Johnston Atoll. The scarcity of endemic species has previously been noted among many different taxa at Johnston Atoll, including fish (Gosline, 1955; Randall et al., 1985; Kosaki et al., 1991), corals (Maragos \& Jokiel, 1986), algae (Buggeln \& Tsuda, 1969; Agegian \& Abbott, 1985; Tsuda et al., 2010) and crustaceans (Edmondson et al., 1925; Amerson \& Shelton, 1976). The only potential endemic species from Johnston Atoll are three unidentified reef fish (Randall et al., 1985; Kosaki et al., 1991), the angelfish Centropyge nahackyi (Kosaki, 1989), the ostracods Parasterope pacifica and Bruuniella beta (Kornicker \& Harrison-Nelson, 2005), the red alga Neotenophycus ichthyosteus (Tsuda et al., 2010), the cyanobacterium Borzia elongata (Tsuda et al., 2010), the gammaproteobacterium Pseudoxanthomonas kalamensis (Harada et al., 2006) and the three unidentified sponges recorded during this study (Table 1). However, several of these species will likely be found in other locations in the future, either as solitary waifs from Johnston Atoll (Kosaki, 1989), or as species that were originally described from Johnston Atoll (Fowler \& Ball, 1925; Bryan, 1926), but were subsequently found to have reproducing populations elsewhere in the Indo-Pacific.

Despite its extreme geographical isolation, the very low level of endemism indicates that Johnston is relatively well connected with other parts of the Pacific, particularly Hawai'i (Grigg, 1981; Grigg et al., 1981; Rivera et al., 2004, 2011; Kobayashi, 2006; Timmers et al., 2011). Kobayashi (2006) used computer simulations and high-resolution current data to identify at least two corridors connecting Johnston Atoll with the Hawaiian Archipelago, an interpretation that is consistent with genetic studies (Rivera et al., 2004, 2011; Timmers et al., 2011). Additionally, many other studies highlight the strong floral and faunal similarities between Johnston Atoll and the Hawaiian Islands (Gosline, 1955; Buggeln \& Tsuda, 1969; Bailey-Brock, 1976; Grigg, 1981; Grigg et al., 1981; Randall et al., 1985; Maragos \& Jokiel, 1986; Kosaki et al., 1991; Coles et al., 2001; Maragos et al., 2004; Tsuda et al., 2010). These studies report that the flora and fauna of Johnston Atoll are mostly composed of species that are also found in Hawai' $i$, with $91 \%$ of all marine species found at Johnston also occurring in Hawai'i (reviewed by Coles et al., 2001). Our results are consistent with these previous studies, as $96 \%$ of species identified during our mesophotic surveys are also known from Hawai' $i$ 
(Tables 1 \& 2). Additionally, this pattern was consistent amongst various taxonomic groups identified during our mesophotic surveys, as $100 \%$ of echinoderms, $99 \%$ of reef fish, $87 \%$ of corals and $78 \%$ of macroalgae are also known to occur in Hawai $i$ (Tables 1 \& 2). In comparison, previous studies noted that $94 \%$ of reef fish species (Randall et al., 1985; Kosaki et al., 1991), 74\% of coral species (Maragos et al., 2004) and $94 \%$ of algal species (excluding cyanobacteria) of Johnston Atoll are also found in Hawai'i (Roy Tsuda, unpublished data). It is currently unknown whether the three unidentified sponge species recorded during this study also occur in Hawai' $i$ and elsewhere in the Indo-Pacific. Further investigations will be necessary to determine the taxonomic status and geographical affinities of the sponge species recorded during our surveys.

Coral reef ecosystems below the depth limits of conventional SCUBA diving remain scarcely surveyed worldwide, and particularly in remote locations like Johnston Atoll. This study represents the first dedicated effort to characterize the mesophotic flora and fauna at Johnston Atoll. Our results support the strong connectivity between Johnston Atoll and the Hawaiian Archipelago highlighted by previous studies. Finally, this study adds 30 new records to Johnston Atoll, thereby emphasizing the value of deep-diving technologies in surveying the largest portion of the depth range of coral reef ecosystems $(30-150 \mathrm{~m})$, which remains largely unexplored.

\section{ACKNDWLEDGEMENTS}

We thank the captain and crew of the NOAA ship 'Hi'ialakai', and J. Leonard, B. Hauk, K. Gleason, G. McFall, D. Pence, J. Copus, K. Lopes, J. Cousins, J. Halonen, B. Prescott, C. Chang and J. Bostick for field support. Special thanks to D. Turner, F. Lichowski and C. Kelley for help with mapping and dive site selection. This work was funded by NOAA's Office of National Marine Sanctuaries, through the Papahānaumokuākea Marine National Monument. The scientific results and conclusions, as well as any views or opinions expressed herein, are those of the authors and do not necessarily reflect the views of NOAA or the Department of Commerce.

\section{REFERENCES}

Agegian C.R. and Abbott I.A. (1985) Deep water macroalgal communities: a comparison between Penguin Bank (Hawaii) and Johnston Atoll. Proceedings of the Fifth International Coral Reef Congress, Tahiti 5, 47-50.

Amerson A.B. and Shelton P.C. (1976) The natural history of Johnston Aoll, Central Pacific Ocean. Atoll Research Bulletin 192, 1-479.

Bailey-Brock J.H. (1976) Habitats of tubicolous polychaetes from the Hawaiian Islands and Johnston Atoll. Pacific Science 30, 69-81.

Boehlert G.W., Watson W. and Sun L.C. (1992) Horizontal and vertical distributions of larval fishes around an isolated oceanic island in the tropical Pacific. Deep-Sea Research 39, 439-466.

Brainard R., Maragos J., Schroeder R., Kenyon J., Vroom P., Godwin S., Hoeke R., Aeby G., Moffitt R., Lammers M., Gove J., Timmers M., Holzwarth S. and Kolinski S. (2005) The state of coral reef ecosystems of the U.S. Pacific remote island areas. In Waddell J. (ed.) The state of coral reef ecosystems of the United States and Pacific freely associated states: 2005. Silver Spring, MD: NOAA Technical Memorandum NOS NCCOS 11. NOAA/NCCOS Center for Coastal Montoring and Assessment's Biogeography Team, pp. 338-372.

Brock V., Jones R.S. and Helfrich P. (1965) An ecological reconnaissance on Johnston Island and the effects of dredging. Honolulu, HI: University of Hawaii, $90 \mathrm{pp}$.

Brock V., Van Heukelem W. and Helfrich P. (1966) An ecological reconnaissance on Johnston Island and the effects of dredging. Honolulu, HI: University of Hawaii, 56 pp.

Brock V.E. and Chamberlain T.C. (1968) A geological and ecological reconnaissance off western Oahu, Hawaii, principally by means of the research submarine 'Asherah'. Pacific Science 22, 373-394.

Bryan E.H. (1926) Insects of Hawaii, Johnston Island and Wake Island. Bernice P. Bishop Museum Bulletin 31, 1-94.

Buggeln R.G. and Tsuda R.T. (1969) A record of benthic marine algae from Johnston Atoll. Atoll Research Bulletin 120, 1-20.

Chave E.H. and Malahoff A. (1998) In deeper waters: photographic studies of Hawaiian deep-sea habitats and life-forms. Honolulu: University of Hawai'i Press.

Chave E.H. and Mundy B.C. (1994) Deep-sea benthic fish of the Hawaiian Archipelago, Cross Seamount, and Johnston Atoll. Pacific Science 48, 367-409.

Chilson L.M. (1953) Insect records from Johnston Atoll. Proceedings, Hawaiian Entomological Society 15, 81-84.

Clark A.H. (1949) Ophiuroidea of the Hawaiian Islands. Bishop Museum Bulletin 195, 1-133.

Coles S.L., DeFelice R.C. and Minton D. (2001) Marine species survey of Johnston Atoll, Central Pacific Ocean, June 2000. Bishop Museum Technical Report 19, 1-64.

Edmondson C.H., Fisher W.K., Clark H.L., Treadwell A.L. and Cushman J.A. (1925) Marine zoology of the tropical central Pacific. Bishop Museum Bulletin 27, 1-148.

Fowler H.W. and Ball S.C. (1925) Descriptions of new fishes obtained by the Tanager Expedition of 1923 in the Pacific Islands west of Hawaii. Proceedings of the Academy of Natural Sciences of Philadelphia 76, 269-274.

Gosline W.A. (1955) The inshore fauna of Johnston Island, a Central Pacific atoll. Pacific Science 9, 442-480.

Grigg R.W. (1981) Acropora in Hawaii. Part 2. Zoogeography. Pacific Science 35, 15-24.

Grigg R.W., Polovina J.J., Friedlander A.M. and Rohmann S.O. (2008) Biology of coral reefs in the Northwestern Hawaiian Islands. In Riegl B.M. and Dodge R.E. (eds) Coral reefs of the USA. Heidelberg: Springer, pp. 573-594.

Grigg R.W., Wells J.W. and Wallace C. (1981) Acropora in Hawaii. Part 1. History of the scientific record, systematics, and ecology. Pacific Science 35, 1-13.

Harada R.M., Campbell S. and Li Q.X. (2006) Pseudoxanthomonas kalamensis sp. nov., a novel gammaproteobacterium isolated from Johnston Atoll, North Pacific Ocean International Journal of Systemic and Evolutionary Microbiology 56, 1103-1107.

Hoover J.P. (2006) Hawai i's sea creatures-a guide to Hawai i's marine invertebrates (revised edition). Honolulu, Hawai i: Mutual Publishing.

Huisman J.M., Abbott I.A. and Smith C.M. (2007) Hawaiian reef plants. Honolulu, Hawaii: University of Hawaii Sea Grant College Program.

Jokiel P.L. and Tyler W.A. (1992) Distribution of stony corals in Johnston Atoll lagoon. Proceedings of the 7 th International Coral Reef Symposium 2, 683-692. 
Kahng S.E., Copus J.M. and Wagner D. (2014) Recent advances in the ecology of mesophotic coral ecosystems (MCEs). Current Opinion in Environmental Sustainability 7, 72-81.

Kahng S.E., Garcia-Sais J.R., Spalding H.L., Brokovich E., Wagner D., Weil E., Hinderstein L. and Toonen R.J. (2010) Community ecology of mesophotic coral reef ecosystems. Coral Reefs 29, 255-275.

Kahng S.E. and Kelley C.D. (2007) Vertical zonation of megabenthic taxa on a deep photosynthetic reef $(50-140 \mathrm{~m})$ in the Au'au Channel, Hawaii. Coral Reefs 26, 679-687.

Kahng S.E. and Maragos J.E. (2006) The deepest, zooxanthellate scleractinian corals in the world? Coral Reefs 25, 254.

Kane C., Kosaki R.K. and Wagner D. (2014) High levels of mesophotic reef fish endemism in the Northwestern Hawaiian Islands. Bulletin of Marine Science 90, 693-703.

Keating B. (1985) Submersible observations on the flanks of Johnston Island (Central Pacific Ocean). Proceedings of the Fifth International Coral Reef Congress 6, 413-418.

Kobayashi D.R. (2006) Colonization of the Hawaiian Archipelago via Johnston Atoll: a characterization of oceanographic transport corridors for pelagic larvae using computer simulation. Coral Reefs 25, 407-417.

Kohler K.E. and Gill S.M. (2006) Coral Point Count with Excel extensions (CPCe): a Visual Basic program for the determination of coral and substrate coverage using random point count methodology. Computers \& Geosciences 32, 1259-1269.

Kornicker L.S. and Harrison-Nelson E. (2005) Ostracoda from Johnston Atoll, Pacific Ocean, and proposal of a new tribe, Bruuniellini (Myodocopina: Cylindroleberididae). Pacific Science 59, 323-362.

Kosaki R.K. (1989) Centropyge nahackyi, a new species of angelfish from Johnston Atoll (Teleostei: Pomacanthidae). Copeia 4, 880-886.

Kosaki R.K., Pyle R.L., Randall J.E. and Irons D.K. (1991) New records of fishes from Johnston Atoll, with notes on biogeography. Pacific Science 45, 186-203.

Lobel P.S. and Lobel L.K. (2008) Aspects of the biology and geomorphology of Johnston and Wake Atolls, Pacific Ocean. In Riegl B.M. and Dodge R.E. (eds) Coral reefs of the USA. Heidelberg: Springer, pp. $655-689$.

Maragos J.E. and Jokiel P.L. (1986) Reef corals of Johnston Atoll: one of the world's most isolated reefs. Coral Reefs 4, 141-150.

Maragos J.E., Potts D.C., Aeby G., Gulko D., Kenyon J., Siciliano D. and VanRavenswaay D. (2004) 2000-2002 rapid ecological assessment of corals (Anthozoa) on shallow reefs of the Northwestern Hawaiian Islands. Part 1: species and distribution. Pacific Science 58, $211-230$.

NOAA (2006) PIFSC cruise report CR-o6-08. Honolulu, Hawaii: NOAA, $100 \mathrm{pp}$.

Olson S.L. (1996) History and ornithological journals of the Tanager expedition of 1923 to the Northwestern Hawaiian Islands, Johnston and Wake Islands. Atoll Research Bulletin 433, 1-210.
Parrish F.A. and Boland R.C. (2004) Habitat and reef-fish assemblages of banks in the Northwestern Hawaiian Islands. Marine Biology 144, $1065-1073$.

Ralston S., Gooding R.M. and Ludwig G.M. (1986) An ecological survey and comparison of bottom fish resource assessments (submersible versus handline fishing) at Johnston Atoll. Fishery Bulletin 84, $141-155$.

Randall J.E. (2007) Reef and shore fishes of the Hawaiian Islands. Honolulu, Hawaii: University of Hawaii Sea Grant College Program.

Randall J.E., Lobel P.S. and Chave E.H. (1985) Annotated checklist of the fishes of Johnston Island. Pacific Science 39, 24-80.

Randall J.E. and Ralston S. (1984) A new species of serranid fish of the genus Anthias from the Hawaiian Islands and Johnston Island. Pacific Science 38, 220-227.

Rivera M.A.J., Andrews A.H., Kobayashi D.R., Wren J.L.K., Kelley C.D., Roderick G.K. and Toonen R.J. (2011) Genetic analyses and simulations of larval dispersal reveal distinct populations and directional connectivity across the range of the Hawaiian Grouper (Epinephelus quernus). Journal of Marine Biology 2011, 11 pp. http:// dx.doi.org/10.1155/2011/765353.

Rivera M.A.J., Kelley C.D. and Roderick G.K. (2004) Subtle population genetic structure in the Hawaiian grouper, Epinephelus quernus (Serranidae) as revealed by mitochondrial DNA analyses. Biological Journal of the Linnean Society 81, 449-468.

Rooney J., Donham E., Montgomery A., Spalding H., Parrish F., Boland R., Fenner D., Gove J. and Vetter O. (2010) Mesophotic coral ecosystems in the Hawaiian Archipelago. Coral Reefs 29, $361-367$.

Smith R. and Swain J. (1882) Notes on a collection of fishes from Johnston's Islands, including descriptions of five new species. Proceedings of the United States National Museum 5, 119-143.

Spalding H.L. (2012) Ecology of mesophotic macroalgae and Halimeda kanaloana meadows in the main Hawaiian Islands. Honolulu, Hawaii: University of Hawaii.

Timmers M.A., Andrews K.R., Bird C.E., deMaintenton M.J., Brainard R.E. and Toonen R.J. (2011) Widespread dispersal of the crown-of-thorns sea star, Acanthaster planci, across the Hawaiian Archipelago and Johnston Atoll. Journal of Marine Biology, $10 \mathrm{pp}$. http://dx.doi.org/10.1155/2011/934269.

and

Tsuda R.T., Abbott I.A., Vroom P.S. and Fisher J.R. (2010) Marine benthic algae of Johnston Atoll: new species records, spatial distribution, and taxonomic affinities with neighboring islands. Pacific Science 64, 585-605.

Correspondence should be addressed to: D. Wagner NOAA, Papahānaumokuākea Marine National Monument 1845 Wasp Boulevard, Building 176, Honolulu, HI 96818 USA email: Daniel.Wagner@noaa.gov 